
Jürgen Popp

\title{
Raman Spectroscopy to Characterize Bladder Tissue for Multidimensional Diagnostics of Cancer in Urology
}

\begin{abstract}
Fiber optic Raman spectroscopy offers labelfree identification of cancer in the bladder under in vivo conditions. However, state-of-the-art Raman technology does not enable to scan the entire bladder wall. Our multidimensional approach within the project Uro-MDD combines panoramic 3D-image reconstruction of white light cystoscopy and fluorescence lifetime imaging to define regions of interest for Raman-assisted diagnostics. First Raman results are presented from human control and cancer bladder specimens that demonstrated how to obtain specific molecular information. Such Raman images can be used in a clinical setting to determine cancer margins and the resection status. Fiber probes are under development to translate the technique to in vivo screening.
\end{abstract}

Keywords: Raman spectroscopy, Raman imaging, endoscopy, cancer diagnosis, chemometric data analysis.

https://doi.org/10.1515/cdbme-2020-3063

\section{Introduction}

Raman spectroscopy is based on inelastic scattering of monochromatic light which probes vibrations of molecular bonds. A spectrum of all Raman-active vibrations is generated in a label-free and non-destructive way and provides a specific signature of analytes. Raman imaging combines the spectroscopic and lateral information. In the context of biomedicine, Raman spectra have been shown to distinguish between normal and pathological cells and tissues, and Raman images give contrast to delineate margins between different tissues down to the cellular level [1]. Raman spectroscopy can also be combined with fiber-optic probes for minimal invasive and intraoperative assessment of tissues [2,3]. One of the main disadvantages of Raman spectroscopy for biomedical

${ }^{*}$ Corresponding author: C. Krafft: Leibniz IPHT, Albert-EinsteinStr. 9, 07745 Jena, Germany, e-mail: christoph.krafft@leibnizipht.de. S. Guo, T. Bocklitz, J. Popp. Leibniz IPHT, Jena,

P. Bronsert, A. Miernik, Medical Center - University of Freiburg. applications is weak signal intensities. This requires usually exposure times in the range of seconds per spectrum which leads to slow image acquisition. Therefore, Raman spectroscopy is often combined with rapid pre-screening techniques to define points of interest. Within the project Uro-MDD, the entire bladder wall is visualized by panoramic 3D-image reconstruction of white light cystoscopy[4] and fluorescence lifetime imaging [5] to define regions of interest for Raman assisted diagnostics. Coherent Raman scattering (CRS) such as coherent anti-Stokes Raman scattering and stimulated Raman scattering offer faster image acquisition with scanning speed in the range of megapixel per second. However, such high speed can only be achieved for single vibrational bands, and transmission of high intensity picosecond laser pulses for CRS excitation by fiber optics requires special fibers which is one of the unmet needs in translational biophotonics [6].

Raman spectroscopy for bladder cancer identification has already been reported in previous papers. A fiber-optic Raman system analyzed 29 bladder tissue samples in an ex vivo setting [7]. The authors developed a classification model to distinguish benign specimens (normal and cystitis) and malignant specimens (transitional cell carcinoma) with an accuracy of $84 \%$. Another study measured Raman maps from cryosections of bladder tissues [8]. A classification model differentiated average Raman spectra from cancer and noncancer of 15 patients with an accuracy of $93 \%$. A high volume fiber-optic Raman probe was used to determine the invasiveness of bladder cancer under in vivo conditions [9]. Raman spectra were collected with exposure times between 1 and 5 seconds from suspicious and non-suspicious regions. An algorithm discriminated bladder cancer and normal bladder with a sensitivity of $85 \%$ and a specificity of $79 \%$. The authors reported that the probe collected mainly signal from deeper tissue layers whereas early changes in the upper urothelial layer were not detected. A recent study collected Raman images from 67 bladder biopsies of 28 patients using a fiber-probe system [10]. After extensive pre-processing to suppress high tissue autofluorescence in Raman spectra, noncancer tissue, low-grade and high-grade cancer were classi- 
fied with an accuracy of $92 \%$ for cancer identification and $84 \%$ for cancer grading.

The current work presents Raman images from cancer and non-cancer specimens of resected bladders. The microscopic setup used a water immersion objective lens to collect high quality Raman spectra that could be analyzed without pre-processing. Detailed molecular and morphological information were obtained from four samples of two patients to characterize bladder tissue.

\section{Methods}

Panoramic imaging, stereo cystoscopy and fluorescence lifetime imaging have been described for bladder cancer detection in an accompanying paper by Wittenberg et al. at this conference. The current paper focuses on sample preparation, Raman spectroscopy and statistical data analysis.

\subsection{Sample preparation}

Control and cancer specimens were prepared from ten human bladder resections. Their sizes ranged from ca. 5-10 mm. Samples were placed in a sample chamber and covered with a calcium fluoride window to protect them from drying. Photographs of patient samples 1 and 2 are shown in Fig. 1. Parallel cyrosections were prepared on calcium fluoride windows for Raman and infrared microspectroscopic imaging. Consecutive sections were stained with hematoxylin and eosin for histopathologic assessment as goldstandard. The liquid around the samples (white in frozen state) is optimal cutting temperature (OCT) medium in which the specimens were immersed before storage in the tissue bank. Control tissue appears red whereas cancer tissue has a brighter hue. Of particular interest is cancer 2 because the bright region in the center is surrounded by a red region.

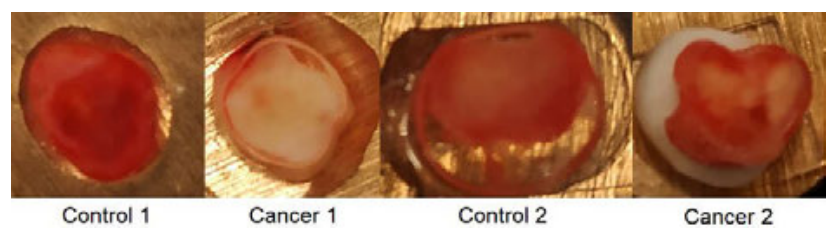

Figure 1: Control and cancer tissue from human bladder resections of patient 1 and 2.

\subsection{Raman spectroscopy}

The Raman spectrometer RXN1 microprobe with a $785 \mathrm{~nm}$ laser (Kaiser Optical Systems, USA) was used for data acquisition. A water immersion objective lens $20 \times /$ NA 0.5 (Zeiss,
Germany) focussed ca. $130 \mathrm{~mW}$ intensity onto the sample. Raman images were registered with an exposure time of 5 seconds per spectrum in a sequential acquisition mode at 250 $\mu \mathrm{m}$ step size. The instrument was controlled by the software Holo-GRAMS which automatically performs laser wavelength calibration, wavenumber calibration and intensity calibration (Kaiser Optical Systems, USA).

\subsection{Data Processing}

Raman images were imported to Matlab (Mathworks, USA) using the toolbox HoloMAP (Kaiser Optical Systems, USA). Without further preprocessing, Raman data were decomposed by the hyperspectral unmixing algorithm called vertex component analysis (VCA). Briefly, VCA calculates endmember loadings and scores that represent the spectra and concentrations of the most dissimilar components. Details of the implementation of in-house scripts have been described elsewhere [11].

\section{Results}

Raman endmember spectra of control and cancer tissue with the score plots as inset are shown in Fig. 2. The Raman spectra coincide for each class with only small variations in background slope, total and relative band intensities. Most Raman bands of control tissues are assigned to spectral contributions of proteins with $\alpha$ helical structures as evident from bands at 934, 1270 and $1655 \mathrm{~cm}^{-1}$. The other labeled bands are assigned to aromatic and aliphatic amino acid residues. The score plots display that that this component is abundant in the right part of control 1 and in the central part of control 2. The Raman spectra of cancer tissue are also dominated by spectral contributions of proteins, however with different secondary structures as evident from more intense and shifted bands at 922, 938, 1246 and $1665 \mathrm{~cm}^{-1}$. These changes together with typical bands of proline and hydroxyproline at 815 and $855 \mathrm{~cm}^{-1}$ point to elevated content of fibrous proteins [12]. The score plots of cancer tissue show a homogeneous distribution in specimen 1 and high abundance only in the upper portion which is consistent with the photograph in Fig. 1.

More tissue components are shown in figure 3. In the center of control specimen 1 lipid-rich regions are detected which are evident from bands in the Raman endmember spectrum (blue trace) at $1748(\mathrm{C}=\mathrm{O}), 1656(\mathrm{C}=\mathrm{C}), 1268$ $(\mathrm{C}=\mathrm{CH})$ and 1440 and $1302 \mathrm{~cm}^{-1}\left(\mathrm{CH}_{2}\right.$ groups $)$ typical for fatty acid chains. Raman spectra with high lipid content were also reported in other bladder studies [9]. The Raman endmember spectrum (green) of cancer specimen 2 is very 
similar to the previous spectrum which indicates lipid-rich regions next to cancer tissue.

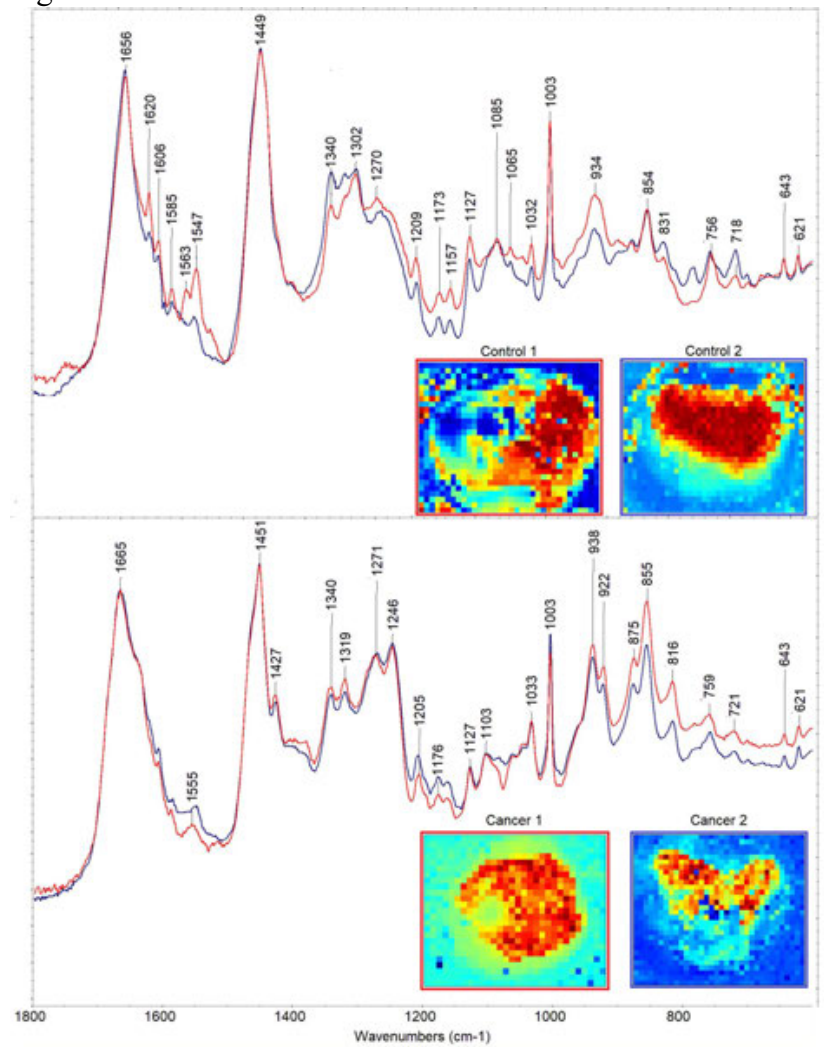

Figure 2: Vertex component analysis of Raman images of bladder specimens. Endmember spectra and score plots (insets) are shown for control (top) and cancer tissue (bottom). Trace colors of spectra coincide with box colors of score plots. Abundances in score plots are scaled from high (red) to low (blue).

Spectral contributions of lipids and OCT medium overlap in the Raman endmember spectrum (red) of cancer specimen 1 as evident from most intense OCT bands near 1121 and 851 $\mathrm{cm}^{-1}$. The VCA algorithm cannot decompose both components because they co-localize throughout the sample. The Raman spectrum of copper phthaloyanine only appeared at one position. This pigment is used as drug for photodynamic therapy (PDT). As the Raman bands are strongly enhanced due to the resonance Raman effect (ca. 200,000 counts compared to 2,000 to 4,000 counts for the other spectra), nonresonance enhanced tissue bands are not visible anymore. As this molecule was not expected, the Raman spectral library Know-It-All (Bio-Rad, USA) was used for identification. Endmember Raman spectra that were not shown for brevity are assigned to substrate background, water between objective lens and substrate window, and OCT. These components did not contribute to differentiation between cancer and noncancer. With one exception (overlap of lipid and OCT), these confounding components were successfully separated from the main tissue constituents proteins and lipids.

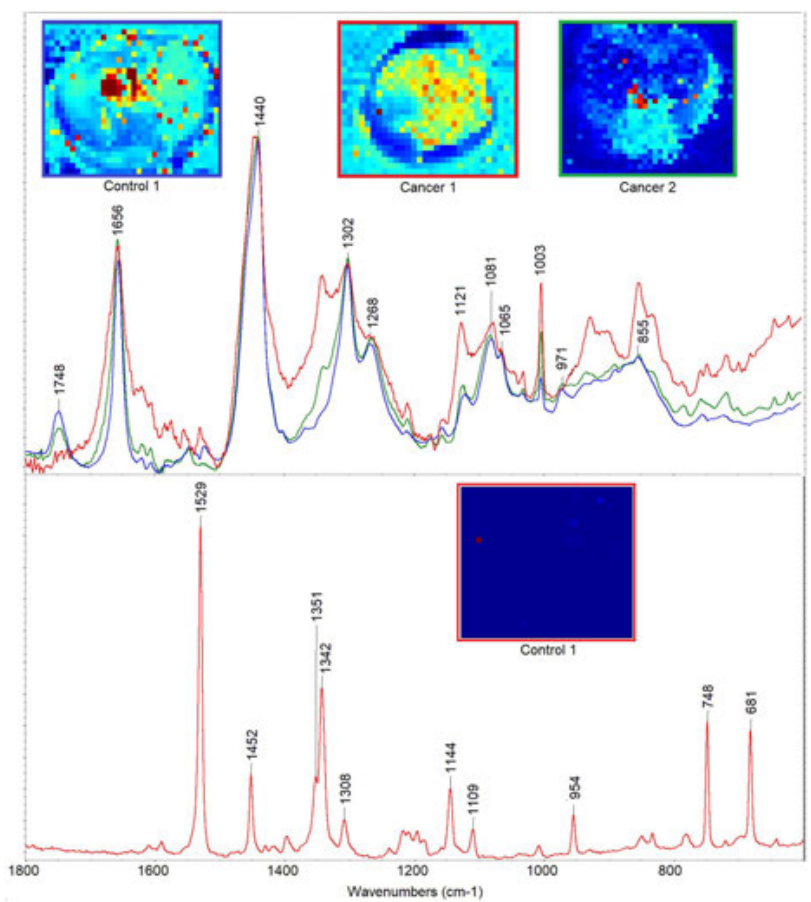

Figure 3: Vertex component analysis of Raman images of bladder specimens. Endmember spectra and score plots are shown for lipid-rich tissues (top) and the PDT drug copper phthalocyanine (bottom). Trace colors of spectra coincide with box colors of score plots. Abundances in score plots are scaled from high (red) to low (blue).

\section{Discussion and Conclusion}

Raman images of bladder specimens were processed by VCA that provided molecular and quantitative information without a priori knowledge or labels, and in an unsupervised way. Four data sets from two patients were described here. The autofluorescence background was remarkably low and the signal-to-noise was exceptionally high in all spectral images which enabled to distinguish normal from diseased tissue, lipid inclusions and a PDT drug. The detection of the drug is consistent with PDT treatment of bladder cancer which induced necrosis. In fact, elevated Raman bands of fibrous proteins in cancer tissues in figure 2 rather point to necrotic tissue than to cancer when compared to another study [10]. 16 additional data sets were collected from eight patients with identical parameters and are expected to give more details about bladder tissue features. Together with reference information of the pathological goldstandard, the Ramanbased information constitutes the basis for supervised classification approaches. This strategy was demonstrated for grading of glioma brain tumors [13]. In Bergner et al. hyperspectral unmixing defined reference Raman spectra of proteins, lipids, cholesterol, cholesterol ester and nucleic acids 
that were used to quantify their abundance in Raman images by non-negativity linear least square fitting and correlate chemical and morphological features with the tumor grade. Other classification algorithms are also applied in the context of Raman tissue imaging such as linear discriminant analysis, support vectors machines or machine learning concepts using artificial intelligence and neural networks $[1,14]$. Even more independent data are required to train and validate robust classification models which could be achieved by a network of research laboratories. These ex vivo results may be transferred to in vivo, intraoperative procedures after Raman instruments are coupled to fiber optic probes and ethical approval is obtained. The state of the art in Raman fiber optic probes was summarized [2,15] and dedicated probes for integration with cystoscopy are under development. The Raman probes have a small diameter of less than $3 \mathrm{~mm}$ which enable to insert them into the working channel of cystoscopes. The video channel and the reconstructed panoramic image will guide the Raman probe to points of interest and the Raman-based assessment will guide the surgical resection.

\section{Author Statement}

Conflict of interest: Authors state no conflict of interest. Informed consent: Informed consent has been obtained from all individuals included in this study.

Ethical approval: The research related to human use complies with all the relevant national regulations, institutional policies and was performed in accordance with the tenets of the Helsinki Declaration, and has been approved by the authors' institutional review board or equivalent committee.

\section{References}

[1] Krafft C, Schmitt M, Schie I, Cialla-May D, Matthaeus C, Bocklitz T, Popp J (2017) Label-free molecular imaging of biological cells and tissues by linear and non-linear Raman spectroscopic approaches. Angewandte Chemie International Edition 56 (16):4392-4430. doi:10.1002/anie.201607604

[2] Cordero E, Latka I, Matthäus C, Schie I, Popp J (2018) In vivo Raman spectroscopy: from basics to applications. Journal of Biomedical Optics 23 (7):071210
[3] Krafft C, von Eggeling F, Guntinas-Lichius O, Hartmann A, Waldner MJ, Neurath MF, Popp J (2018) Perspectives, potentials and trends of ex vivo and in vivo optical molecular pathology. Journal of Biophotonics 11 (1):e201700236. doi:10.1002/jbio.201700236

[4] Kriegmair MC, Bergen T, Ritter M, Mandel P, Michel MS, Wittenberg T, Bolenz C (2017) Digital Mapping of the Urinary Bladder: Potential for Standardized Cystoscopy Reports. Urology 104:235-241.

doi:https://doi.org/10.1016/j.urology.2017.02.019

[5] Becker W (2019) The bh TCSPC handbook. 8th edn. Becker \& Hickl, Berlin

[6] Krafft C, Popp J (2019) Medical needs for translational biophotonics with the focus on Raman-based methods. Translational Biophotonics 1 (1-2):e201900018. doi:10.1002/tbio.201900018

[7] Crow P, Molckovsky A, Stone N, Uff B, Wilson BC, Wong Kee Song LM (2005) Assessment of fiber-optic near infrared Raman spectroscopy for diagnosis of bladder and prostate cancer. Urology 65:1126-1130

[8] de Jong BW, Schut TC, Maquelin K, van der Kwast T, Bangma CH, Kok DJ, Puppels GJ (2006) Discrimination between nontumor bladder tissue and tumor by Raman spectroscopy. Anal Chem 78 (22):7761-7769

[9] Draga RO, Grimbergen MC, Vijverberg PL, van Swol CF, Jonges TG, Kummer JA, Ruud Bosch JL (2010) In vivo bladder cancer diagnosis by high-volume Raman spectroscopy. Anal Chem 82 (14):5993-5999

[10] Cordero E, Rüger J, Marti D, Mondol AS, Hasselager T, Mogensen K, Hermann GG, Popp J, Schie IW (2020) Bladder tissue characterization using probe-based Raman spectroscopy: Evaluation of tissue heterogeneity and influence on the model prediction. Journal of Biophotonics 13 (2):e201960025. doi:10.1002/jbio.201960025

[11] Hedegaard M, Matthäus C, Hassing S, Krafft C, Diem M, Popp J (2011) Spectral unmixing and clustering algorithms for assessment of single cells by Raman microscopic imaging. Theor Chem Acc 130 (4-6):1249-1260

[12] Krafft C, Codrich D, Pelizzo G, Sergo V (2008) Raman and FTIR microscopic imaging of colon tissue: a comparative study. J Biophoton 1 (2):154-169

[13] Bergner N, Medyukhina A, Geiger KD, Kirsch M, Schackert G, Krafft C, Popp J (2013) Hyperspectral unmixing of Raman micro-images for assessment of morphological and chemical parameters in non-dried brain tumor specimens. Analytical and Bioanalytical Chemistry 405 (27):8719-8728. doi:10.1007/s00216-013-7257-7

[14] Pradhan P, Guo S, Ryabchykov O, Popp J, Bocklitz TW (2020) Deep learning a boon for biophotonics? Journal of Biophotonics 13(6):e201960186. doi:10.1002/jbio.201960186

[15] Krafft C, Dochow S, Latka I, Dietzek B, Popp J (2012) Diagnosis and screening of cancer tissues by fiber optic Raman spectroscopy. Biomedical Spectroscopy and Imaging 1:39-55 